\title{
Entanglement generation and simultaneity with superconducting qubits
}

\author{
Carlos Sabín \\ Instituto de Física Fundamental, CSIC, Serrano, 113-bis 28006 Madrid (Spain) \\ csl@iff.csic.es
}

\begin{abstract}
We analyse DCE-entanglement between two superconducting qubits in several states of qubit motion. We show that correlated absorption and emission of photons is crucial, which can be linked to the special-relativity notion of simultaneity. (c) 2018 The Author(s)
\end{abstract}

OCIS codes: $270.5580,270.5585,350.4010$

Parametric amplification of a multimode superconducting cavity have been recently proved to be a useful source of bipartite and multipartite mode entanglement [1]. A particular instance is the well-known case of the dynamical Casimir effect (DCE), where the parametric amplification is provided by a non-adiabatic modulation of the boundary conditions, which turns into two-mode-squeezed entangled mode pairs [2] generated out of the quantum-field vacuum. This mode entanglement can be also swapped to a pair of superconducting qubits, where each of them interacts with a single cavity mode in a separated pair of cavities sharing a common wall which undergoes DCE modulation [3].

An important practical advantage of superconducting circuit architectures is the wide tunability of the parameters, which allows them not only to reproduce quantum-optical schemes in the microwave regime, but also to reach unexplored regimes. This is particularly interesting in the context of analogue quantum simulations. Indeed, the DCE can be seen as an instance of this approach, since it is the possibility of ultrafast modulation of the external magnetic flux threading a SQUID which enables the implementation of mirror-like boundary conditions moving at relativistic speeds. Along similar lines, related quantum field theoretical effects such as the Unruh effect - the measurement of thermal radiation by a detector uniformly accelerated through the quantum vacuum- can also be considered in these setups. In particular, a single-mode version of the Unruh effect -called "cavity-enhanced Unruh effect" or simply "acceleration radiation"- was introduced by Scully and collaborators in 2004 [4-7]. In a quantum-optics setup, this would be just a gedanken experiment, due to the impossibility of achieving the required accelerations. However, in this talk we will see that it is possible to realize an analogue quantum simulation in a superconducting quantum-circuit architecture.

We will show that the modulation of the qubit-cavity coupling strength can mimic the variation of the coupling strength of a two-level atom traversing an optical cavity as in Scully's proposal. Coupling tunability is a feature of several superconducting qubits. In particular, we choose a modified transmon architecture, where the tunability is also provided by a SQUID, as in the DCE. As a result of the simulated motion, acceleration radiation appears [8], giving rise to interesting features such as entanglement generation and non-trivial modifications of the emission properties [9]. Moreover, even effective superluminal qubit motion can be simulated, giving rise to a new form of radiation, which is a simulated Ginzburg radiation [10]. It is natural then to ask what are the effects of this virtual qubit motion in the generation of entanglement by means of the DCE. We analyse this by adding to the setup of [3] the extra ingredient of coupling tunability in order to simulate the qubit motion. We find [11] that generic motion would break down the synchronization in the absorption and emission of the DCE pair by the qubits, resulting in a dramatic decrease of entanglement, as compared to the scenario where the qubits are static. Only qubit trajectories which respect the synchronized absorption and emission of photons preserve the high degree of entanglement generated in [3]. Besides the technological applications as entanglement source, this scheme suggests an interesting link with the notion of simultaneity in special relativity, opening the path to quantum simulations of textbook gedanken experiments conceived by Einstein.

\section{References}

1. Sandbo Chang, C.W, Simoen,M., Aumentado, J., Sabín, C., Forn-Diaz,P., Vadiraj,A. M., Quijandría, F., Johansson, G., Fuentes, I., Wilson, C.M., Generating Multimode Entangled Microwaves with a Superconducting Parametric Cavity Phys. Rev. Applied 10, 044019 (2018).

2. Wilson,C. M., Johansson, G., Pourkabirian, A., Simoen, M., Johansson, J. R., Duty, T., Nori, F. and Delsing, P., Observation of the Dynamical Casimir Effect in a superconducting circuit Nature 479, 376 (2011). 
3. Felicetti, S.,Sanz, M., Lamata, L., Romero, G., Johansson, G., Delsing, P. and Solano, E., Dynamical Casimir Effect Entangles Articial Atoms, Phys. Rev. Lett. 113093602 (2014).

4. Scully, M. O., Kocharovsky, V. V., Belyanin, A. , Fry, E. and Capasso, F. Enhancing acceleration radiation from ground-state atoms via cavity quantum electrodynamics Phys. Rev. Lett. 91, 243004 (2003).

5. Hu, B. L. and Roura, A. Comment on "Enhancing acceleration radiation from ground-state atoms via cavity quantum electrodynamics" Phys. Rev. Lett. 93, 129301 (2004).

6. Scully, M. O., Kocharovsky, V. V., Belyanin, A., Fry, E. and Capasso, F., Scully et al. Reply Phys. Rev. Lett. 93, 129302 (2004).

7. Belyanin, A., Kocharovsky, V. V., Capasso, F., Fry, E., Zubairy, M. S. and Scully, M. O. Quantum electrodynamics of accelerated atoms in free space and in cavities Phys. Rev. A 74, 023807 (2006).

8. Felicetti, S., Sabín, C., Fuentes, I., Lamata, L., Romero, G. and Solano, E. Relativistic motion with superconducting circuits Phys. Rev. B 92, 064501 (2015).

9. García-Álvarez, L., Felicetti, S., Rico, E., Solano,E. and Sabín, C. Entanglement of superconducting qubits via acceleration radiation Sci. Rep. 7, 657 (2017).

10. Sabín, C., Peropadre, P., Lamata, L. and Solano, E., Simulating superluminal physics with superconducting circuit technology Phys. Rev. A 96, 032121 (2017).

11. Agustí, A., Solano, E., Sabín, C., Effects of qubit motion on DCE-generated entanglement (in preparation) 